\title{
Management of Pathergy in Pyoderma Gangrenosum: A Case Report \& Literature Review
}

\author{
Md Mydin Siddik NSF1,2*, Lai Kuan C², Chor Lip HT33, Mohd Yussof \\ $S^{1,2}$ and Ibrahim $S^{2}$ \\ ${ }^{1}$ Plastic and Reconstructive Surgery, Universiti Teknologi MARA, Malaysia \\ 2Plastic and Reconstructive Surgery, Hospital Sungai Buloh, Malaysia \\ ${ }^{3}$ Pusat Perubatan Universiti Kebangsaan, Malaysia
}

Case Report

Volume 3 Issue 3

Received Date: October 17, 2018

Published Date: November 23, 2018

DOI: $10.23880 /$ cdoaj-16000161

*Corresponding author: Dr Nur Shazwani Farah Md. Mydin Siddik, Department of Plastic \& Reconstructive Surgery, UITM Medical Faculty, Jalan Hospital, 47000, Sungai Buloh, Selangor, Malaysia, Tel: (00603) 61265004; Fax: (00603) 61265224; Email: drshazwanifarah@gmail.com

\section{Abstract}

Pyoderma Gangrenosum(PG) is uncommon with only with a global incidence of 3-10 cases per mil- lion per year. PG exhibits pathergy, which is the development or worsening of a lesion secondary to trauma or surgery. Due to the limited number of cases, the management of pathergy in PG is lacking in the literatures. In this article, a middle aged woman of Malay descend suffering from ulcerative colitis presented for local wound care of a PG complicated with infection and ulceration. The objective of this report is to emphasize on the modalities used in treating the pathergy of PG.

Keywords: Pyoderma gangrenosum; Pathergy; Ulcerative colitis; Plastic surgery

Abbreviations: PG: Pyoderma Gangrenosum; RCTs: Randomized Controlled Trials; IBD: Inflammatory Bowel Disease.

\section{Introduction}

Pyoderma gangrenosum (PG) is a non-infectious, inflammatory neutrophilic dermatosis typically characterized by necrotizing ulceration and is often triggered from minor trauma or surgery, a condition known as pathergy. PG can occur alone or associated with systemic diseases. A meta-analysis found that more than half of patients with PG present with an underlying systemic disease.
PG is frequently misdiagnosed due to the lack of definitive laboratory or histopathological diagnostic criteria. Typical histological evaluation shows epidermal ulceration and sterile neutrophilic infiltration without vasculitis or without granuloma formation. The nonspecific histological features pose a great challenge in the diagnosis and only made after excluding other pathologies.

There is no concensus on the treatment of PG with the current treatment targeting on reducing inflammation through anti-inflammatory and immunosuppressive agents. Pathergy can occur in $30 \%$ of patients with PG. Successful surgical treatment has been reported when the disease is in quiescent after immunosuppressive therapy. 


\section{Case Presentation}

A 46 years old female with background illness of ulcerative colitis was referred by the dermatology team for the management of pyoderma gangrenosum over the right thigh. The wound started as a small blister with purulent discharge which was unresponsive to antibiotics. Examination revealed a large ulcer measuring $8 \times 10 \mathrm{~cm}$ over the lateral aspect of the right thigh with erythematous edges (Figure 1A). A skin swab culture grew Pseudomonas Aeruginosa. Skin biopsy was also performed and showed moderate to marked dermal neutrophilic and histiocytic infiltrates, with no evidence of malignancy, fungal or tuberculosis infection. She was treated for PG with Pseudomonas Aeruginosa infection. High dose intravenous steroids and a broad spectrum antibiotic that was organism-sensitive were administered in the ward.

Regular dressing was done for the first two weeks and the wound became less exudative (Figure 1B). However, the wound was found to be infected during her clinic visit and two other similar lesions appeared adjacent to the wound (Figure 1C). A decision was made for conservative surgical debridement of necrotic tissue. Post-operatively, antibiotics were administered and wounds were dressed with non-adherent dressing. Topical steroids were applied when the wounds were clean (Figures 2A \& 2B). After 3 months of dressings and immunosuppressive medication, the wound subsequently epithelialized (Figure 2C).

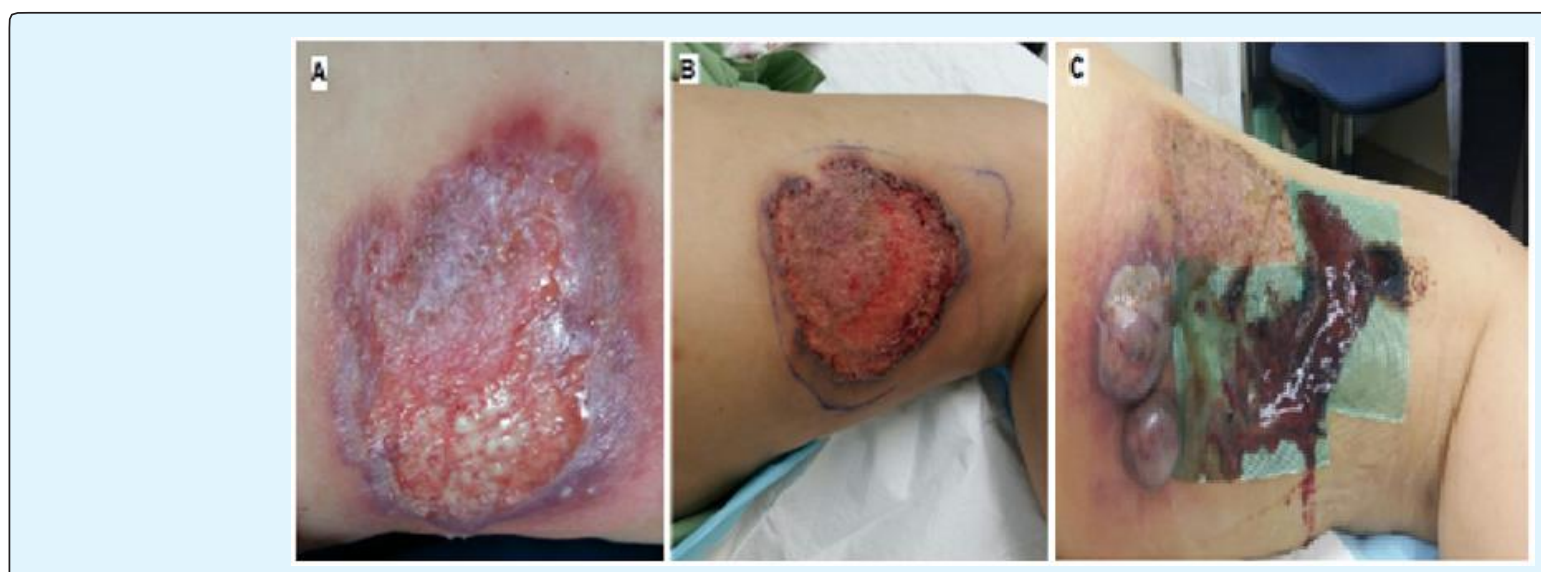

Figure 1: 1A: Day 0 - Original infected PG ulcer, treated with super-oxidized disinfectant solution covered with paraffin gauze impregnated with chlorhexidine acetate $0.5 \%$.

1B: Day 13- wound is healing with healthy granulation tissue seen.

1C: Day 24- wound is re-infected with 3 new surrounding lesions (green arrow). This wound was treated with special coated dressing with hydrophobic properties with external polyurethane foam.
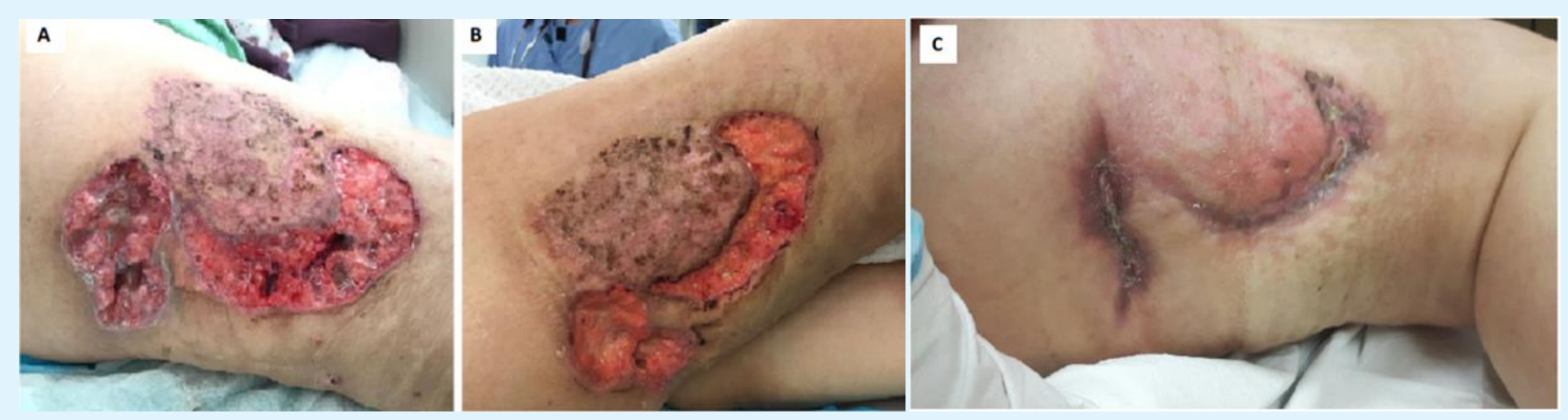

Figure 2: 2A: Day 42 - There is keratinization of original wound (green arrow), dressing was changed to polyester mesh impregnated with hydrocolloid, petroleum jelly and silver sulphadiazine particles.

2B: Day 54 - Gradual contraction is seen and dressing was continued with special coated dressing with hydrophobic properties.

2C: Day 100 - Full contraction and keratinization of the original PG ulcer. 

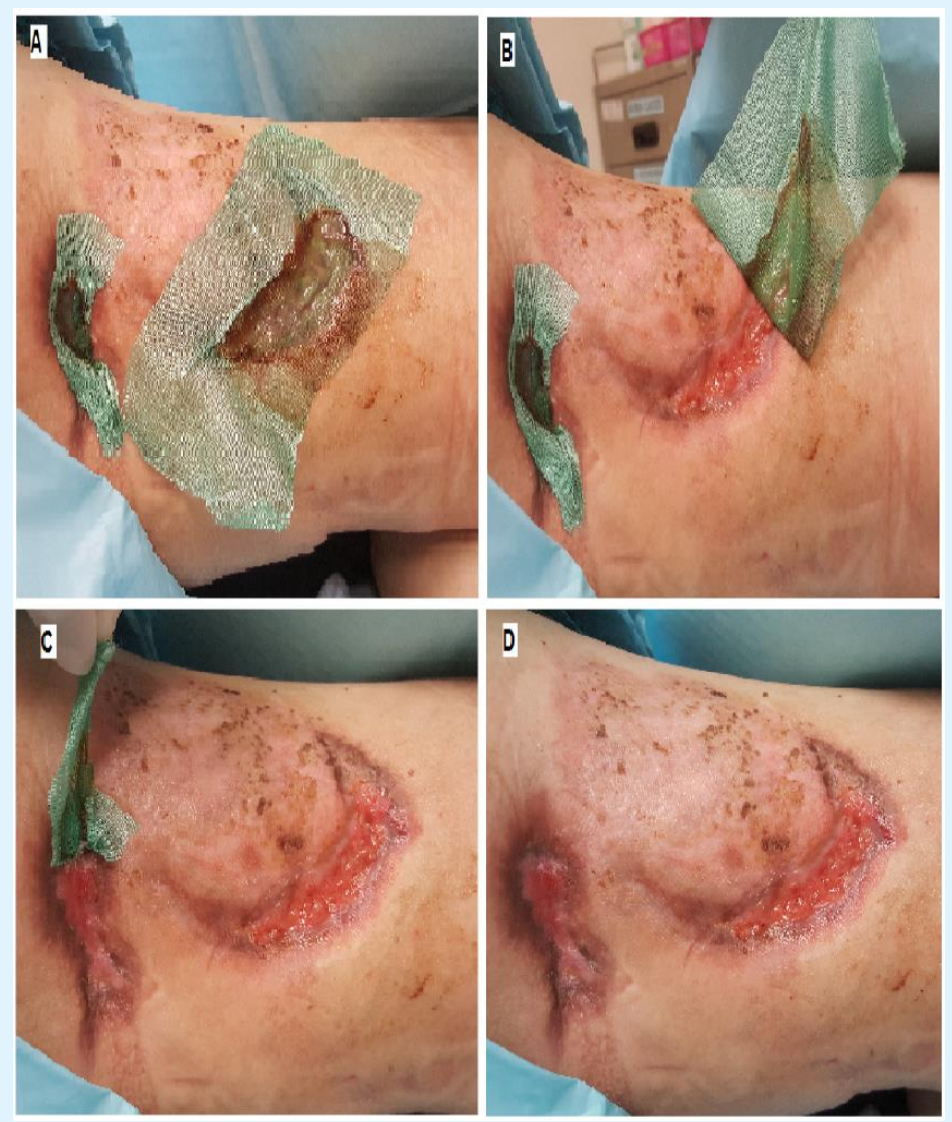

Figure 3: A: Special coated dressing with hydrophobic properties. B\&C: Gentle removal with minimal adhesiveness reduces pain on dressings. D: Complete removal of dressings.

\section{Discussion}

Pyoderma Gangrenosum is an extraintestinal cutaneous manifestation of IBD which encompasses Ulcerative Colitis and Crohn's Disease that occurs in only $0.37 \%$ of the population [1,2]. The pathogenesis of $P G$ is still poorly understood with a primary treatment of long term immunosupression and local wound care [3].

The objective of this case report is to focus on the management of pathergy in PG done by our plastic surgical unit. PG ulcers classically presents as a deep ulcer with a well defined bluish border as seen in this patient and represent as a 'cat paw' appearance [3]. The ulcer edge is often worn and the surrounding skin is erythematous and indurated. Ulcers with pathergic lesions develop at the site of minor trauma, so surgery or debridement is contraindicated [4]. The epidemiology of Pyoderma is estimated to have an incidence rate of 3-10 per million population per year $[5,6]$. Complicated by the underlying systemic disease and immunosuppressive treatment, wound healing may take up till six months for epithelialization. Surgery is avoided unless the wound is infected with active exudation to prevent pathergy associated worsening [4].

There is limitation of literature because the exact prevalence has not been systematically reviewed. These ulcers exhibit pathergy which means with trauma, the wound will worsen or develop new wounds. Debridement of ulcers suspicious of PG was thought to cause more new lesion due to possible pathergy phenomenon. However, there are reported cases with successful skin grafting when combined with systemic corticosteroid or immunosuppressant.

Management should involve treatment of infection with antibiotics and immunosuppression therapy. For early disease, topical steroids or Tacrolimus can be used. For aggressive disease, oral Prednisolone $0.5-1 \mathrm{mg} / \mathrm{kg}$ per day tapered over 3 months is first line treatment. Cyclosporin and Azothioprine are reserved for second and 
thirdline therapies. Other options include other immunosuppressive drugs, intravenous immunoglobulin, and targeted therapies such as anti-TNF $\alpha$, anti-interleukin 1 , antiinterleukin $12,23[7,8]$.

Reported that approximately $57 \%$ of patients with PG presented with underlying systemic disease, which IBD being the leading associated disease, followed by arthritis (12.8\%), hematological and solid malignancies (8.9\%). The prevalence of the pathergy phenomenon was $16.3 \%$. The pathergy phenomenon has previously been reported about $30 \%$ with pre-existing PG $[9,10]$.

PG is very difficult to treat, and reported in association of systemic treatments combined with a correct wound bed preparation approach [6]. The fundamental approach is to control the associated condition. Anti-inflammatory and immunosuppressive drugs can be effective both in PG and the underlying disease, as the example of the IL1 antagonist anakinra for PG in the context of PAPA syndrome, and of infliximab and other anti-TNF agents in Crohn's disease and ulcerative colitis. Among the anti-TNF biologics, etanercept is reported not effective in IBD.

Surgical intervention alone can worsen PG condition if it is associated with pathergy. The modalities related to surgical intervention can be performed in conjunction with immunosuppressant. A noninvasive procedure such as allograft was suggested to avoid pathergy. Other surgical approaches have been described, such as the use of negative pressure wound therapy associated with splitthickness skin grafting and adequate immunosuppression [9]. A combination therapy of allograft and anti-TNF $\alpha$ can improve a graft successful rate with a reduction in immune response against the allograft.

Corticosteroids, immunosuppressive (ciclosporin) and biologics (infliximab, adalimumab) have been successfully used in the treatment of PG. Randomized controlled trials (RCTs) showed complete healing rate of $15-20 \%$ within 6 weeks. However, in patients with concurrent inflammatory bowel disease (IBD), biologics demonstrated $100 \%$ healing rate [11]. Although systemic corticosteroids are considered as first line treatment, small PG (less than $2 \mathrm{~cm}$ square) may benefit from topical or intralesional corticosteroids when used on the active border of the ulcer [12]

Recent genetic studies suggested that both PG and its syndromic form, PASH (pyoderma gangrenosum, acne, suppurative hidradenitis) are a spectrum of polygenic autoinflammatory condition, with similar immunological profiles and mutation in several autoinflammatory genes.
The understanding of immunological and genetic basis in PG, in particular the IL-1 and IL-17, could shed light for possible targeted therapy in the future treatment modalities [13].

\section{Conclusion}

A multimodalities approach is key to a successful treatment of Pyoderma Gangrenosum. Treatment involves appropriate immunosuppressants and control of infection. Meticulously applied dressings that inflict minimal trauma and friction is important to prevent pathergy.

\section{References}

1. Tan WC, Ong CK, Kang SC, Leong KN (2007) Pyoderma Gangrenosum. Med J Malaysia 62(3): 251253.

2. Sani AD, Vora DS (2006) Pyoderma Gangrenosum: A Systemic Review of the Incidence and Prevalence. Journal for Clinical Studies 7(1): 42-45.

3. Brooklyn T, Dunnill Giles, Probert C (2006) Diagnosis and treatment of pyoderma gangrenosum. BMJ 333(7560): 181-184.

4. Ghersi MM, Ricotti C, Nousari CH, Newman MI (2007) Negative pressure dressing in the management of pyoderma gangrenosum ulcer. Arch Dermatol 143(10): 1249-1251.

5. Schultz GS, Barillo DJ, Mozingo DW, Chin GA, Wound Bed Advisory Board Members (2004) Wound bed preparation and a brief history of TIME. Int Wound J 1(1): 19-32.

6. Angelis B, Lucarini L, Agovino A, Migner A, Orlandi F, et al. (2013) Combined use of superoxidised solution with negative pressure for the treatment of pressure ulcers: case report. Int Wound J 10(3): 336-339.

7. Ulkür E, Oncül O, Karagöz H, Celiköz B, Cavuşlu S (2005) Comparison of silver-coated dressing (Acticoat), chlorhexidine acetate $0.5 \%$ (Bactigrass), and silver sulfadiazine 1\% (Silverdin) for topical antibacterial effect in Pseudomonas aeruginosacontaminated, full-skin thickness burn wounds in rats. J Burn Care Rehabil 26(5): 430-433.

8. Wasiak J, Cleland H, Campbell F, Spinks A (2013) Dressings for superficial and partial thickness burns. Cochrane Database Syst Rev 28(3): CD002106. 


\section{Clinical Dermatology Open Access Journal}

9. Choi JS, Lee JH, Kim SM, Kim YJ, Choi JY, et al. (2015) Hydrogel-impregnated dressings for graft fixation: a case series. J Wound Care 24(7): 326-328.

10. Harding K, Gottrup F, Jawień A, Mikosiński J, Twardowska-Saucha K, et al. (2012) A prospective, multi-centre, randomised, open label, parallel, comparative study to evaluate effects of AQUACEL $\AA$ Ag and Urgotul ${ }^{\circledR}$ Silver dressing on healing of chronic venous leg ulcers. Int Wound J 9(3): 285-294.

11. Partridge ACR, Bai JW, Rosen CF, Walsh SR, Gulliver WP, et al. (2018) Effectiveness of systemic treatments for pyoderma gangrenosum: A systematic review of observational studies and clinical trials. Br J Dermatol 179(2): 240-241.

12. Alavi A, French LE, Davis MD, Brassard A, Kirsner RS (2017) Pyoderma Gangrenosum: An Update on Pathophysiology, Diagnosis and Treatment. Am J Clin Dermatol 18(3): 355-337.

13. Marzano AV, Damiani G, Ceccherini I, Berti E, Gattorno M, et al. (2017) Autoinflammation in pyoderma gangrenosum and its syndromic form (pyoderma gangrenosum, acne and suppurative hidradenitis). Br J Dermatol 176(6): 1588-1598.

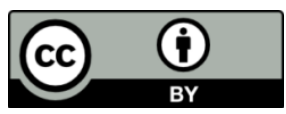

